\title{
Expression Pattern of Early Transcription Factors in Porcine Oocytes and Embryos
}

\author{
So Yeon Kim, Tao Lin, Joo Bin Lee, Jae Eun Lee, Hyun Young Shin and Dong Il Jin* \\ Division of Animal and Dairy Sciences, Chungnam National University, Daejeon 34134, Korea
}

Received June 17, 2019

Revised June 18, 2019

Accepted June 27, 2019

\author{
*Correspondence \\ Dong II Jin \\ Division of Animal and Dairy Science, \\ Chungnam National University, 99 Daehak- \\ ro, Yuseong-gu, Daejeon 34134, Korea \\ Tel: +82-42-821-7854 \\ Fax: +82-42-821-5771 \\ E-mail: dijin@cnu.ac.kr \\ ORCID \\ https://orcid.org/0000-0001-6586-4393
}

\begin{abstract}
Many transcription factors are involved in directing the growth of porcine oocytes. The localization and expression level of a given transcription factor often differ at each stage of early embryonic growth, which spans from fertilization to the formation of the blastocyst. A hallmark of the blastocyst stage is the separation of the endodermal and mesodermal ectoderm. The embryo's medium and its effects are known to be crucial during early development compared to the other developmental stages, and thus require a lot of caution. Therefore, in many experiments, early development is divided into the quality of oocyte and cumulus cells and used in experiments. We thought that we were also heavily influenced by genetic reasons. Here, we examined the expression patterns of five key transcription factors (CDX2, OCT4, SOX2, NANOG, and E-CADHERIN) during porcine oocyte development whose expression patterns are controversial in the pig to the literature. Antibodies against these transcription factors were used to determine the expression and localization of them during the early development of pig embryos. These results indicate that the expressions of key transcription factors are generally similar in mouse and pig early developing embryos, but NANOG and SOX2 expression appears to show speciesspecific differences between pig and mouse developing embryos. This work helps us better understand how the expression patterns of transcription factors translate into developmental effects and processes, and how the expression and localization of different transcription factors can crucially impact oocyte growth and downstream developmental processes.
\end{abstract}

Keywords: cumulus cells, porcine embryos, porcine oocytes, transcription factors

\section{INTRODUCTION}

The growth of germ cells is regulated by many molecules, and the acquisition of meiotic ability and meiotic maturation guarantees the pluripotency of an oocyte. Transgenic animal production takes advantage of accumulating research evidence regarding the growth and development of the oocyte. The development of blastocysts is largely divided into early embryonic development and late embryonic development. Early embryonic development, which spans from the 1-cell stage to the early blastocyst stage, can be observed following parthenogenetic activation (PA). Numerous studies have focused on early development in mice, and many involved transcription factors have been identified. However, far less is known about the transcription factors associated with pig development. Here, we tested whether some of the key transcription factors associated with early development in 
mice (CDX2, OCT4, SOX2, NANOG, and E-CADHERIN) could be involved in early development of oocyte and embryo to confirm the previous work in pig system.

CDX2 is reportedly expressed in primate blastocysts and the late-dividing 2-cell embryos in mice (Deb et al., 2006). OCT 4 has been shown to influence embryonic versatility in mice. It is reportedly expressed in mouse oocytes (Scholer et al., 1989; Rosner et al., 1990; Yeom YI et al., 1991; Palmieri et al., 1994) and has been shown to be essential for forming the inner cell mass (ICM) and to affect the retention of pluripotency of blastomeres (Nichols et al., 1998). The localization patterns of OCT4 were found to be consistent in human and cow embryos (Degrelle et al., 2005); in mice, it is expressed throughout the early embryo up to the blastocyst stage, whereupon it becomes localized to the ICM (Palmieri et al., 1994). NANOG is involved in the self-renewal of undifferentiated embryonic stem cells. NANOG transcripts were reportedly found in early embryos of several stages, including the 4-cell, 8-cell, morula, and blastocyst stages (Brevin et al., 2007; Kumar et al., 2007; Blomberg et al., 2008; Hall et al., 2009). In mouse blastocysts, NANOG expression is reportedly restricted to the ICM (Chazaud et al., 2006; Silver et al., 2009.) The localizations of OCT4 and NANOG have been shown to differ significantly between mammalian species during embryonic development (Kirchhof et al., 2000; Kuijk et al., 2008). Bovine blastocysts OCT4 and NANOG showed up at the same time in the ICM (Degrelle et al., 2005).

SOX2 is known to form a complex with OCT4 to aid in the regeneration of ICM pluripotency. In mouse embryos, SOX2 is expressed during the early developmental stages, such as during the development of oocytes, and in the primitive ectoderm and embryonic lobe (Avilion et al., 2003). SOX 2 plays crucial roles in maintaining pluripotency of mouse embryonic stem cells (Masui et al., 2007). It is essential for embryonic development, is the earliest known marker for embryonic cells prior to the formation of the ICM (Guo et al., 2010), and contributes to proper formation of the ICM (Avilion et al., 2003). SOX2 modulates the expression of NANOG, which mediates various pluripotency factors, including OCT4 (Rodda et al., 2005). During mouse embryonic development, SOX 2 was partially detected in some blastomeres of 4-cell embryos (Keramari et al., 2010). However, it was observed that ICM of blastocysts was expressed in most blastomeres (Avilion et al., 2003). SOX2 is also known to affect the development of bovine embryos (Khan et al., 2012). In bovine embryos, SOX2 is first expressed at the early morula stage; in blastocysts, it is expressed in the ICM but excluded from the trophectoderm (TE).

E-CADHERIN was known to be first observed at the 2 -cell stage in various mammalian species, and undergo phosphorylation around the 8-cell stage, then and cause densification of morula. In many mammals, E- CADHERIN is known to critically contribute to blastocyst formation by mediating cell-cell interactions. In mouse embryos, Ecadherin has been shown to play an important role in the establishment of blastocysts (Larue et al., 1994; Ohsugi et al., 1997). During gastrulation, E-CADHERIN is expressed in the ectoderm and endoderm, but not in the mesoderm (Damjanov et al., 1986; Burdsal et al., 1993; Ciruna et al., 2001). In sheep embryos, E-CADHERIN is confined to the cytoplasm in early embryo and appears at the junctions of 8- to 16-cell embryo (Modina et al., 2010).

Here, we used antibodies to determine the expression and localization of CDX2 and OCT4 during the early development of pig embryos, and compared the obtained data to the results previously obtained in mouse embryos. Also, we examined whether NANOG and SOX2 are expressed in early developing pig embryo by imaging and localization studies. The expression and localization of CDX2, OCT4, NANOG and SOX2 in pig embryo were analyzed to examine how their expression patterns might differ from previous results obtained using mouse embryos. We also examined the expression and localization of ECADHERIN during the early development of porcine embryos.

\section{MATERIALS AND METHODS}

\section{Materials}

All chemicals used in this study were purchased from Sigma-Aldrich (St Louis, MO, USA) unless otherwise specified.

\section{Oocyte collection and maturity}

Porcine ovaries were obtained from a local slaughterhouse. Within $1 \mathrm{hr}$ of collection, the ovaries were transported to the laboratory in saline solution at $37^{\circ} \mathrm{C}$. Follcle size of 3-6 $\mathrm{mm}$ in diameter were collected along with at least three layers of cumulus cells, and the cumulus- 
oocyte complexes were cultured in TCM 199 medium containing 1\% PFF (porcine follicular fluid), 0.01\% PMSG (pregnant mare serum gonadotropin), 0.01\% HCG (human chorionic gonadotropin), $0.001 \%$ EGF (epidermal growth factor), and $0.005 \%$ vitamins. The cells were cultured for $24 \mathrm{hr}$ at $37.5^{\circ} \mathrm{C}$ in a $5 \% \mathrm{CO}_{2}$ containing incubator.

\section{Parthenogenetic (PA)}

PZM3 $(500 \mathrm{~mL} /$ well) topped with $300 \mathrm{~mL} /$ well of mineral oil was distributed to a 4-well dish. In vitro-matured oocytes were washed three times with PZM3, washed three times with mannitol, and then subjected to electric shock (1100 vol/sec, ELECTRO CELL MANIPULATOR ECM2001, BMS, Seoul, Republic of Korea). The oocytes were washed three times in PZM3 and incubated in a 4-well dish at $37.5 \mathrm{oC}$ in $5 \% \mathrm{CO}$. Each PA-derived embryo was checked daily for $\sim 7$ days. PA-derived embryos at various developmental stages (1-cell, 2-cell, 4-cell, 8-cell, morula and blastocyst; $n=10$ per stage) were subjected to immunohistochemical staining.

\section{Fixation and immunostaining}

In vitro-matured oocytes were washed three times in PBG (PVA/PBS) at $27^{\circ} \mathrm{C}$, fixed for $30 \mathrm{~min}$ in $2 \%$ paraformaldehyde in PBS using a Danching shake DS400 (BMS, Seoul, Republic of Korea), and then permeabilized for 30 min in PBS containing 0.5\% Triton X-100 and $100 \mathrm{mM}$ glycine The oocytes or embryos were then incubated for $10 \mathrm{~min}$ in 3\% BSA, and finally washed with PBG for $5 \mathrm{~min}$. Immunostaining was performed with primary antibodies against the following: CDX2 (1:1000, rabbit monoclonal EPR2764Y; Abcam, Seoul, Republic of Korea), OCT-3/4 (C-10) (1:1000, sc5279), NANOG (1:1000, mouse monoclonal), SOX2 (1:1000, mouse monoclonal), and E-CADHERIN (1:1000, mouse monoclonal) (all from Santa Cruz Biotechnology, Santa Cruz, CA). The utilized second antibodies included: goat anti-mouse SC-2092 (red; used for OCT4, SOX2, NANOG, and E-CADHERIN) and mouse anti-rabbit sc-2359 (green; used for CDX2) (both from Santa Cruz Biotechnology). The oocytes were incubated overnight with the primary antibody, washed with PBG for 20 min, and then incubated with the appropriate secondary antibody in the dark at $26^{\circ} \mathrm{C}$ for $1 \mathrm{hr}$. The chromatin was stained with DAPI (4,6-diamidino-2-phenylindole), and the samples were stored at $4{ }^{\circ} \mathrm{C}$ in a slide box until imaging was performed using conforcal microscopy (ZEISS,
Germany).

\section{RESULTS}

\section{Expression of OCT4 and CDX2 in porcine oocytes and embryos}

We used immunohistochemical staining to observe the expression and localization patterns of OCT4 and CDX2 in pig oocyte and embryo ranging from the germinal vesicle (GV) stage (immature oocytes) to the blastocyst stage. DAPI was used to show the entire chromatin. At the GV stage, OCT4 was strongly expressed in the surround nucleus (SN) chromatin and weakly expressed throughout the cytoplasm, while CDX2 was weakly expressed throughout the cytoplasm (Fig. 1). 1-cell stage was stained after 20 hours of PA. At the 1-cell stage, CDX2 and OCT4 were expressed in the cytoplasm. One day later, at the 2-cell stage, CDX2 was expressed in the cytoplasm and OCT4 was strongly expressed in the nuclei. At the 3-cell stage, CDX2 was weakly expressed in the cytoplasm and nuclei, while OCT4 was more prominently expressed in both regions. OCT4 and CDX2 were weakly expressed in the cytoplasm and nuclei at the later stage. In blastocysts, OCT4 was found to be expressed in nuclei of cells around ICM, whereas CDX2 was more ubiquitously expressed throughout the embryo.

\section{Expression of NANOG in porcine oocytes and embryos}

In GV-stage oocytes, NANOG was weakly expressed in the cytoplasm and strongly expressed in the chromatin area (Fig. 2). At the early development stage (1cell, 2-cell, 3-cell, 5-cell and morula) embryos, NANOG was expressed weakly in the cytoplasm. At the blastocyst stage, NANOG was expressed throughout the cytoplasm. In contrast to other mammalian species, nuclear NANOG expression was not detected in developing porcine embryos, and its expression was not confined to the cells of the ICM at the blastocyst stage. Staining was performed for each step.

\section{Expression of SOX2 in porcine oocytes and embryos}

At the GV stage, SOX2 was weakly expressed in the cytoplasm (Fig. 3). At the 1-cell and 2-cell stages, SOX2 was expressed throughout the cytoplasm but absent from the nucleus. At the 4-cell stage, SOX2 was expressed in the nucleus of blastomeres. SOX2 was expressed weakly in the 
cytoplasm at the later stage. At the morula and blastocyst stages, SOX2 was expressed weakly in the cytoplasm and nuclei.

\section{Expression of E-CADHERIN in porcine oocytes and embryos}

At the GV stage oocyte and 1-cell stage embryo, E-CADHERIN was barely expressed in the cytoplasm (Fig. 4). At the later stages, E-CADHERIN was expressed in the cytoplasm but not in the nucleus. E- CADHERIN is well known to be expressed in the cell contact area, but at the 2-cell or 4-cell stages, E-CADHERIN was not observed at the cell wall or cell contact area. At the morula stage, E-CADHERIN was more expressed unevenly in the cytoplasm, whereas at the blastocyst stage, E-CADHERIN was detected at the cell to cell contact area and in the junctions



Fig. 1. The expression patterns of CDX2 (green) and OCT4 (red) in porcine embryos were examined by immunostaining. DAPI (blue) was used to stain DNA. Scale bar $=50 \mu \mathrm{m}$. between cells, as observed in other mammalian species.

\section{DISCUSSION}

We selected four transcription factors known to be involved in the early development of various mammals (CDX2, OCT4, NANOG, and SOX2), and examined their expression patterns during the early developing embryos of pig. In mice, OCT4 and SOX2 affect pluripotency in developing embryos and contribute to forming and maintaining the ICM and CDX2 is expressed from the 2-cell stage, but confined in the TE (Palmieri et al., 1994). OCT4, SOX2, and NANOG have been found to play important roles in early lineage segregation when expressed during early embryonic development (Palmieri et al., 1994; Rodda et al., 2005; Masui et al., 2007; Kuijk et al., 2008).

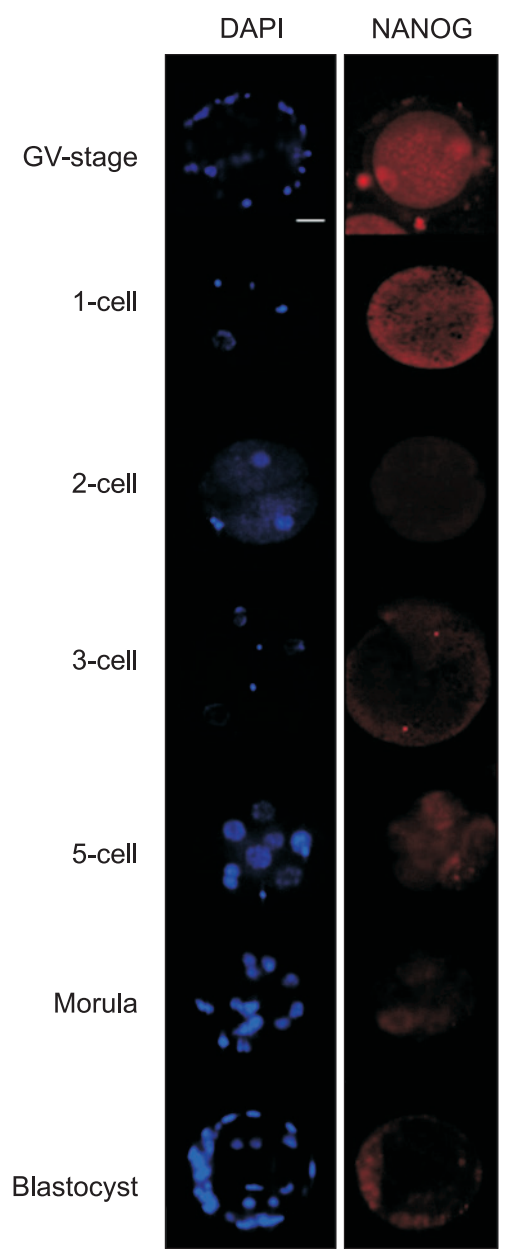

Fig. 2. The expression pattern of NANOG (red) in porcine embryos was examined by immunostaining. DAPI (blue) was used to stain DNA. Scale bar $=50 \mu \mathrm{m}$ 
NANOG critically contributes to the formation of epiblasts and hypoblasts during early mouse embryonic development. Given that CDX2, OCT4, SOX2, NANOG, and E-CADHERIN appear to play major roles in the early embryonic development of mice, we herein used immunochemistry to examine the expression patterns of these factors in porcine oocytes and embryos.

We found that CDX2 was not expressed in GV stage, 1-cell, 2-cell, and morula, but was expressed in blastocyst. Specifically, it was not expressed in the ICM region in blastocyst but was expressed only in TE. OCT4 was stained in the background in GV stage and 1-cell but were strongly expressed in nucleus strongly in 2-cell, and 3-cell and 6-cell. It was strongly expressed in all nuclei in blastocyst. Previous studies have shown that OCT4 is mostly restricted to cells of the ICM in blastocysts (Emura et al.,



Fig. 3. The expression pattern of SOX2 (red) in porcine embryos was examined by immunostaining. DAPI (blue) was used to stain DNA. Scale bar $=50 \mu \mathrm{m}$.
2016), whereas it is expressed in cells of the ICM and TE in mouse blastocysts (Palmieri et al., 1994). In the next experiment, it is necessary to check the exact area by observing the latter blastocyst additionally. Our present results indicate that porcine oocytes and embryos show patterns of CDX2 and OCT4 expression that are similar to those seen in the mouse. These findings, together with the previous reports, suggest that CDX2 and OCT4 are likely to play important roles in the early embryonic development of the pig.

Our analysis of NANOG revealed that it was expressed at all of the studied stages, and was strictly localized in the cytoplasm. It was concentrated in the ICM region during most of the blastocyst stage, and appeared to be expressed in the embryonic disc region during the late hatching stage of the blastocyst. In the mouse, NANOG

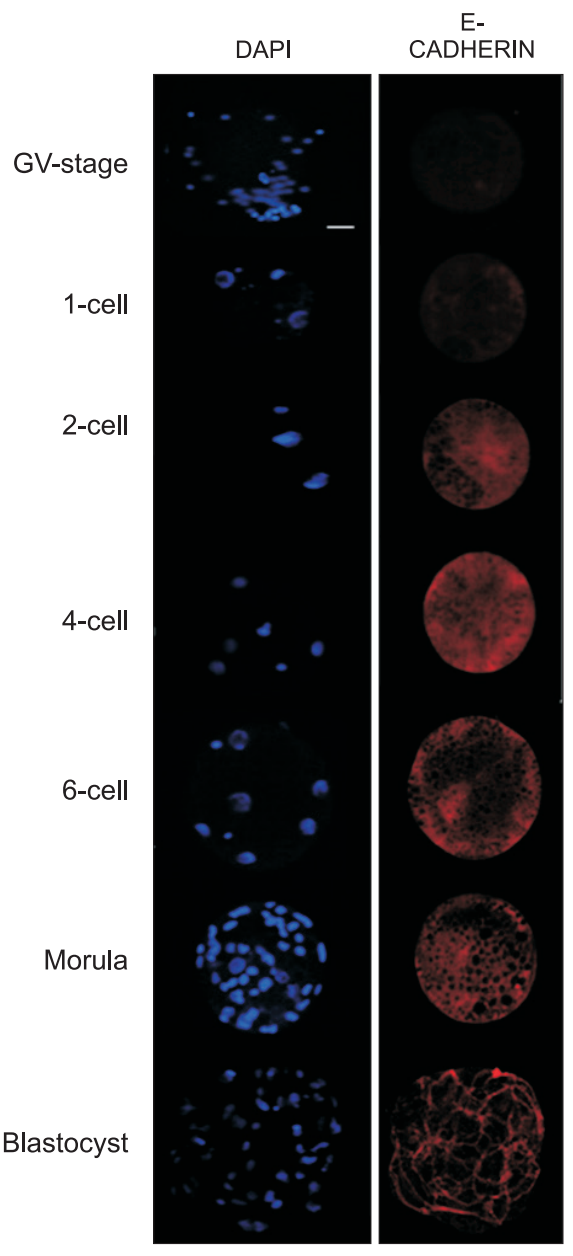

Fig. 4. The expression pattern of E-CADHERIN (red) in porcine embryos was examined by immunostaining. DAPI (blue) was used to stain DNA. Scale bar $=50 \mu \mathrm{m}$. 
was reportedly expressed at the 4-cell, 8-cell, morula, and blastocyst stages (Rodda et al., 2005). Thus, NANOG expression appears to show species-specific differences between pig and mouse developing embryos.

SOX2 is a well-known early embryonic marker that is expressed in the cell nuclei of mouse oocytes and embryos during various developmental stages. However, our analysis revealed that SOX2 was expressed exclusively in the cytoplasm of embryonic pig cells at all tested stages, with the exception of the 4-cell stage, when one of the four nuclei showed detectable expression of SOX2. In particular, SOX2 staining was intense in the ICM area of morula- and blastocyst-stage embryos. SOX2 and NANOG are found in the cytoplasm of early-stage blastocysts, and their expression patterns will appear in later stages.

In the mouse embryo, E-CADHERIN is expressed mainly in the cell wall and cell junction area, suggesting that it plays an important role in early embryonic development (Ohsugi et al., 1997). In porcine blastocysts, we found that E-CADHERIN was expressed specifically near the cell wall area and at the junctions. This indicates that ECADHERIN plays an important role in nuclear division during the early embryonic development of the pig. In other mammals, E-CADHERIN is reportedly expressed at the overlapping part of the cell wall beginning at the 2 -cell stage, which contrasts with our current findings in pig blastocysts. It can be assumed that species-specific differences in the expression of transcription factors during the blastocyst stage can affect the post-blastocyst development.

In sum, we herein show that the expressions of key transcription factors are generally similar in mouse and pig early developing embryos. However, we also identify some species-specific differences. Future work is needed to clarify these differences and their implications, as well as to assess the expression levels of key transcription factors during the later stages of blastocyst development.

\section{CONFLICTS OF INTEREST}

No potential conflict of interest relevant to this article was reported.

\section{ACKNOWLEDGEMENTS}

This study was supported by research funding from
Chungnam National University (2017).

\section{ORCID}

So Yeon Kim: https://orcid.org/0000-0001-8080-3126

Tao Lin: https://orcid.org/0000-0001-9100-6042

Joo Bin Lee: https://orcid.org/0000-0002-6106-9082

Jae Eun Lee: https://orcid.org/0000-0003-1435-5980

Hyun Young Shin: https://orcid.org/0000-0003-4248-7331

Dong Il Jin: https://orcid.org/0000-0001-6586-4393

\section{REFERENCES}

Avilion AA, Nicolis SK, Pevny LH, Perez L, Vivian N and LovellBadge R. 2003. Multipotent cell lineages in early mouse development depend on SOX2 function. Genes Dev. 17: 126140 .

Blomberg LA, Schreier LL and Talbot NC. 2008. Expression analysis of pluripotency factors in the undifferentiated porcine inner cell mass and epiblast during in vitro culture. Mol. Reprod. Dev. 75:450-463.

Brevini TAL, Tosetti V, Crestan M, Antonini S and Gandolfi F. 2007. Derivation and characterization of pluripotent cell lines from pig embryos of different origins. Theriogenology 67:54-63.

Burdsal CA, Damsky CH and Pedersen RA. 1993. The role of ECADHERIN and integrins in mesoderm differentiation and migration at the mammalian primitive streak. Development 118:829-844

Chazaud C, Yamanaka Y, Pawson T and Rossant J. 2006. Early lineage segregation between epiblast and primitive endoderm in mouse blastocysts through the Grb2-MAPK pathway. Dev. Cell 10:615-624

Ciruna B and Rossant J. 2001. FGF signaling regulates mesoderm cell fate specification and morphogenetic movement at the primitive streak. Dev. Cell 1:37-49.

Damjanov I, Damjanov A and Damsky CH. 1986. Developmentally regulated expression of the cell-cell adhesion glycoprotein cell-CAM 12080 in peri-implantation mouse embryos and extraembryonic membranes. Dev. Biol. 116:194-202.

Deb K, Sivaguru M, Yong HY and Roberts RM. 2006. CDX2 gene expression and trophectoderm lineage specification in mouse embryos. Science 311:992-996.

Degrelle SA, Campion E, Cabau C, Reinaud P, Richard C, Renard JP and Hue I. 2005. Molecular evidence for a critical period in mural trophoblast development in bovine blastocysts. Dev. Bio. 288:448-460.

Emura N, Sakural N, Takahashi K, Hashizume T, and Sawal K. 2016. OCT-4 expression is essential for the segregation of trophectoderm lineages in porcine preimplantation embryos. J. Reprod. Dev. 62:401-408.

Guo G, Huss M, Tong GQ Wang C, Sun LL, Clarke ND and Robson P. 2010. Resolution of cell fate decisions revealed by sin- 
gle-cell gene expression analysis from wygote to blastocysts. Dev. Cell 18: 675-685.

Hall VJ, Christensen J, Gao Y, Schmidt MH and Hyttel P. 2009. Porcine pluripotency cell signaling develops from the inner cell mass to the epiblast during early development. Dev. Dyn. 238:2014-2024.

Keramari M, Razavi J, Ingman KA, Patsch C, Edenhofer F, Ward CM and Kimber SJ. 2010. SOX2 is Essential for Formation of Trophectoderm in the Preimplantation Embryo. PLoS One. 5:e13952.

Khan DR, Dube D, Gall L, Peynot N, Ruffini S, Laffont L, Bouthis DL, Degrelle S and Jouneau A. 2012. Expression of Pluripotency Master Regulators during Two Key Developmental Transitions: EGA and Early Lineage Specification in the Bovine Embryo. PLoS one 7: e34110.

Kirchhof N, Carnwath JW, Lemme E, Anastassiadis K, Scholer $\mathrm{H}$ and Niemann H. 2000. Expression pattern of OCT-4 in preimplantation embryos of different species. Biol. Reprod. 63:1698-1705.

Kuijk EW, Puy LD, Van Tol HTA, Oei CHY, Haagsman HP, Colenbrander B and Roelen BAJ. 2008. Differences in early lineage segregation between mammals. Dev. Dyn. 237:918-927.

Kumar BM, Jin HF, Kim JG, Ock SA, Hong Y, Balasubramanian S, Choe SY and Rho GJ. 2007. Differential gene expression patterns in porcine nuclear transfer embryos reconstructed with fetal fibroblasts and mesenchymal stem cells. Dev. Dyn. 236:435-446.

Larue L, Ohsugi M, Hirchenhanin J, and Kemler R. 1994. Ecadherin null mutant embryos fail to form a trophectoderm epithelium. Proc. Natl. Acad. Sci. USA 91:8263-8267.

Masui S, Nakatake Y, Toyooka Y, Shunisato D, Yagi R, Rakahashi K, Okochi H,Okuda A, Matoba R, Sharov AA, Ko MS and Niwa H. 2007. Pluripotency governed by SOX2 via regulation of OCT3/4 expression in mouse embryonic stem cells. Nat. Cell Biol. 9: 625-635.

Medeja ZE, Sosnowski J, Hryniewicz K, Warzych E, Pawlak P, Rozwadowska N, Plusa B and Lechniak D. 2013. Changes in sub-cellular localisation of trophoblast and inner cell mass specific transcription factors during bovine preimplantation development. BMC Dev. Biol. 13;13:32.

Modina S, Leoni GG, Lodde V, Naitana S, Pirani S, Succu S, Berlinguer $\mathrm{F}$ and Luciano AM. 2010. Involvement of E-CADHERIN in early in vitro development of adult and juvenile sheep. Reprod. Fert. Dev. 2:468-477.

Nichols J, Zevnik B, Anastassiadis K, Niwa H, Klewe-Nebenius D, Chambers I, Scholer H and Smith A. 1998. Formation of pluripotent stem cells in the mammalian embryo depends on the POU transcription factor OCT4. Cell 95:379-391.

Ohsugi M, Larue L, Schwarz H, and Kemler R. 1997. Cell-junctional and cytoskeletal organization in mouse blastocysts lacking E-CADHERIN. Dev. Biol. 185:261-271.

Palmieri SL, Peter W, Hess H and Scholer HR. 1994. OCT-4transcription factor is differentially expressed in the mouse embryo during establishment of the first two extraembryonic cell lineages involved in implantation. Dev. Biol. 166;259267.

Rodda DJ, Chew JL, Lim LH, Loh YH, Wang B, Ng HH and Robson P. 2005. Transcriptional regulation of NANOG by OCT4 and SOX2. J. Biol. Chem. 280: 24731-24737.

Rosner MH, Vigano MA, Ozato K, Timmons PA, Poirie R, Rigby PW and Staudt LM. 1990. A Pov-domain transcription factor in early stem cells and germ cells of the mammalian embryo. Nature 345:686-692.

Scholer HR, Hatzopoulos AK, Balling R, Suzuki N and Gruss P. 1989. A family of octamer-specific proteins present during mouse embryogenesis; evidenve for germline-specific expression of an OCT factor. EMBO J. 8:2543-2550.

Yeom YI, Ha HS, Scholer HR and Artzt K. 1991. Structure, expression and chromosomal location of the OCT-4 gene. Mechanisms Dev. 35:171-179.

Yuan H, Corbi N, Basilico C and Dailey L. 1995. Developmental-specific activity of the FGF-4 enhancer requires the synergistic action of SOX2 and OCT-3. Genes Dev. 9:2635-2645. 